\title{
Hepatic clearance of apoptotic lymphocytes: simply removal of waste cells?
}

\author{
A. Chionna, E. Panzarini, P. Pagliara, A. De Luca, S. Caforio, L. Abbro, L. Dini \\ Dept. of Biological and Environmental Science and Technology, University of Lecce, Italy
}

\begin{abstract}
(C)2003, European Journal of Histochemistry
The in situ liver recognition of apoptotic lymphocytes was studied by using different sources of lymphocytes (i.e. human, rat and mouse) and animal models (i.e. rat and mouse). Lymphocytes were induced to apoptosis using $10^{-2} \mathrm{M}$ cycloheximide for up to 24 hours; three types of apoptosing lymphocytes, corresponding to different stages in the apoptotic process, were described: type 1 or early apoptosis, type 2 or mature apoptosis and type 3 or late/necrotic apoptosis. When livers were in situ injected with apoptotic lymphocytes enriched for type 1 (early), 2 (mature) or 3 (late/necrotic) apoptosis, they recognized and internalized apoptosing cells, with an efficiency directly dependent on the stage of the apoptotic process. The highest recognition rate, which was, in all cases, mediated by galactose- and mannose-specific receptors, was obtained with homologous apoptotic cells (i.e. rat lymphocytes and rat liver). Moreover, the drastically reduced efficiency of recognition of human or mouse apoptotic lymphocytes when injected into rat liver, suggested the involvement also of species-specific antigens.
\end{abstract}

Key words: liver sinusoids, apoptosis, lymphocytes, rat, mice, human

Correspondence: Prof. Luciana Dini, Dept. of Biological and Environmental Science and Technology, University of Lecce, via per Monteroni, 73100 Lecce, Italy; Phone: international +39.0832. 320614. Fax. international +39.0832.320626.

E-mail: Iuciana.dini@unile.it

Paper accepted on December 9, 2002

European Journal of Histochemistry 2003; vol. 47 issue 2 [Apr-Jun]:97-104
T he liver is the largest internal organ and the main site for protein synthesis, bile formation, glycogen production and detoxification (Desmet 1994). Moreover, phagocytosis, one of the specific functions of the liver, is performed by the sinusoidal cells (i.e. endothelial and Kupffer cells) (Smedsrot et al. 1990).

Recent studies have revealed that the liver is the main site for apoptosis of blood cells, either as a battlefield or as a graveyard (Sun and Shi 2001; Crispe et al. 2000). The large amount of apoptotic cells in the liver may be responsible for the distinctive characteristics of the role of liver in immunity and its acceptance of grafting (Starzel et al. 1989).Indeed, it is well known that human leukocyte typing in clinical liver transplantation is not as crucial as in the transplantation of other organs, such as the kidney, heart and pancreas (Sun and Shi 2001). Moreover, the maintenance of immune tolerance by apoptosis as described in many experimental systems could explain the liver immune responses (Sun and Shi 2001).

Although the mechanisms concerning the distinct immunological function of the liver remains unclear, the available data suggest that the large number of apoptotic cells that specifically accumulate in the liver are partially responsible for this (Crispe et al. 2000). A preferential accumulation of apoptotic lymphocytes by sinusoidal liver cells has been reported (Dini and Carlà 1998).

The exact identity of the cells responsible for the recognition of apoptotic cells is far from being entirely revealed, although there is more and more evidence to suggest that macrophages are the main scavengers for apoptotic cells. As liver Kupffer cells account for $80 \%$ of all macrophages in mammals, it is reasonable to assume that the liver is a specialized organ for inducing and/or clearing apoptotic cells (Dini 2000; Dini et al. 2002). A great deal is now known, both of the molecules on the surface of apoptotic cells that are responsible for phagocytic 
recognition, and of the receptors involved (Savill 1998; Savill and Fadok 2000; Schlegel and Williamson 2001; Fadok et al. 2001). However, much remains to be established; in particular, in order to understand the tolerance-inducing mechanism, it is important to know which signals are transduced from the phagocyte to the lymphocyte or vice-versa. In addition, correct elimination of apoptotic cells is important to prevent the onset of autoimmune diseases (Rosen and Casciola-Rosen 1999; Rovere et al. 1999). Studies have clearly demonstrated that apoptotic cells are the source of autoantigens, and that insufficient clearance of apoptotic cells results in the accumulation of autoantigens in the circulation, which activates the immunosystem and leads to systemic autoimmunity (Rodenburg et al. 2000; Lorenz et al. 1997).

To better understand the mechanism of apoptotic lymphocyte accumulation in the liver, the recognition of lymphocytes (from various Mammalian species) at different stages of their apoptotic program by the sinusoidal liver cells was studied.

\section{Materials and Methods Cells and treatments}

Cells and cultures. Peripheral mononuclear blood cells were obtained after Ficoll gradient separation of buffy coats from blood donated by non-smoking healthy males, aged 25 to 45 . Peripheral blood lymphocytes (PBL) were separated from monocytes by double adherence to plastic; they were over $95 \%$ pure as judged by morphological criteria. They were maintained at a cell density of $1 \times 10^{6} \mathrm{cell} / \mathrm{s} / \mathrm{mL}$ in complete culture medium at $37^{\circ} \mathrm{C}$ supplemented with $10 \%$ inactivated fetal calf serum (FCS), 2 $\mathrm{mM}$ L-glutamine, $100 \mathrm{IU} / \mathrm{mL}$ pennicillin and streptomycin in a humidified atmosphere of $5 \% \mathrm{CO}_{2}$; cells were used on the first day of explant.

Induction of apoptosis. Apoptosis was induced with $10^{-2} \mathrm{M}$ cycloexhimide ( $\mathrm{CHX}$ ) for up to $24 \mathrm{hrs}$, followed by $\mathrm{lhr}$ of recovery in fresh medium.

\section{Analysis and quantification of apoptosis}

Cell viability was assessed by membrane impermeability to trypan blue and propidium iodide, positive staining with vital dyes, and normal nuclear shape and texture as revealed by vital staining with Hoechst 33342, Hematoxilyn and Eosyn.

Apoptosis was detected by light microscopy and quantified by cytofluorimetry.

Nuclear fragmentation was detected after stain- ing with the vital dye Hoechst 33342 ( $1 \mu \mathrm{g} / \mathrm{mL}$ ) according to the nuclear morphological features. The percentages of both apoptotic and viable cell fractions were determined by cytometry with an EPICS XL cytofluorimeter (Coulter Electronic Inc. Hialegh, $F L, U S A$ ) equipped with a 5-W argon laser having a 488-nm excitation wavelength. The fixed cells were stained with propidium iodide ( 10 $\mu \mathrm{g} / \mathrm{mL}$ ) in phosphate saline buffer containing 40 units $/ \mathrm{mL}$ Rnase and $0.5 \%$ Tween 20. The 635-nm emission wavelength was monitored for propidium iodide emission. For each of the flow cytometry analyses, at least 10,000 events were calculated. The stage of the cell cycle was determined from the amount of incorporated propidium iodide. The percentage of apoptotic cells was based on the sub GI fraction.

\section{Transmission electron microscopy}

The ultrastructure of lymphocytes was obtained by transmission electron microscopy. $10^{6}$ cells $/ \mathrm{mL}$ were fixed with $2.5 \%$ glutaraldehyde in cacodilate buffer, $\mathrm{pH} 7.4$, for $1 \mathrm{hr}$ at ice temperature, postfixed with $1 \% \mathrm{OsO}_{4}$ in the same buffer, dehydrated, embedded in Spurr resin and examined under a Philips CM12 TEM.

\section{Lectins and phosphatydilserine cytochemistry}

Lymphocytes, fixed with $4 \%$ paraformaldehyde in phosphate buffer $\mathrm{pH} 7.4$ for $10 \mathrm{~min}$ and deposited on gelatinated microscopy slides, were analyzed for sugars' surface localization by using ConcanavalinA (Con-A, $40 \mu \mathrm{g} / \mathrm{mL}$, mannose), Helix pomatia ( $\mathrm{HPA}, 60 \mu \mathrm{g} / \mathrm{mL}, \mathrm{N}$-acetylgalctosamina) and U/ex europaeus (UEA, $40 \mu \mathrm{g} / \mathrm{mL}$, $\alpha$-fucose) FITC conjugates for 30 min at room temperature in the dark. AnnexinV-FITC conjugate was used to localize phosphatydilserine on the cell surface; it was used at a concentration of $20 \mu \mathrm{M}$ for $20 \mathrm{~min}$ at room temperature in the dark. Samples were observed with conventional and confocal fluorescent microscopy (PCM 2000 Nikon microscope).

\section{In situ liver injection of apoptotic lymphocytes}

Livers were perfused in a non-recirculating system at a flow rate of $1 \mathrm{~mL} / \mathrm{min} .1 \times 10^{6}$ lymphocytes labelled with Hoechst 33342 were injected into the liver circulation (total volume $10 \mathrm{~mL}$ ). Lymphocytes used in the different experiments were nonapoptotic, or type 1, type 2 or type 3 apoptotic cells. The livers were then extensively washed with 
culture medium to remove unbound cells. Livers, fixed in $4 \%$ paraformaldehyde in phosphate buffer, were processed for routine light microscopy or for cryosectioning. The adhesion of lymphocytes to the sinusoidal wall was evaluated by light and fluorescence microscopy of liver sections. The adhesion specificity was tested in parallel inhibition experiments by adding $80 \mathrm{mM}$ (final concentration) of a sugar cocktail ( $\mathrm{N}$-acetyl-D- glucosamine, $\mathrm{N}$-acetylD- galactosamine, fucose, mannose) into the perfusion tube before lymphocyte administration.

The concentration of lymphocytes used for all experiments was at saturation point, as evaluated by the presence of apoptotic lymphocytes in the medium collected from the liver. Experiments were performed on mice and rats.

\section{Results \\ Liver recognition of apoptotic lymphocytes increases with apoptotic progression}

Peripheral human, rat and mouse blood lymphocytes, were induced to apoptosis by $\mathrm{CHX} 10^{-2} \mathrm{M}$ for up to $24 \mathrm{hrs}$. During the $24 \mathrm{hrs}$ of $\mathrm{CHX}$ incubation, the cells were monitored for their progressive accumulation of cell surface and morphological (nucleus and cell shape) modifications. According to the differences observed, three types of apoptosing lymphocytes were described (Figure 1). The three types of apoptosis were found at all times and irrespective of the species used, during the 24 hrs of incubation with $\mathrm{CHX}$, but with different percentages (Table 1).

Type 1 or early apoptosis. The majority of the cell
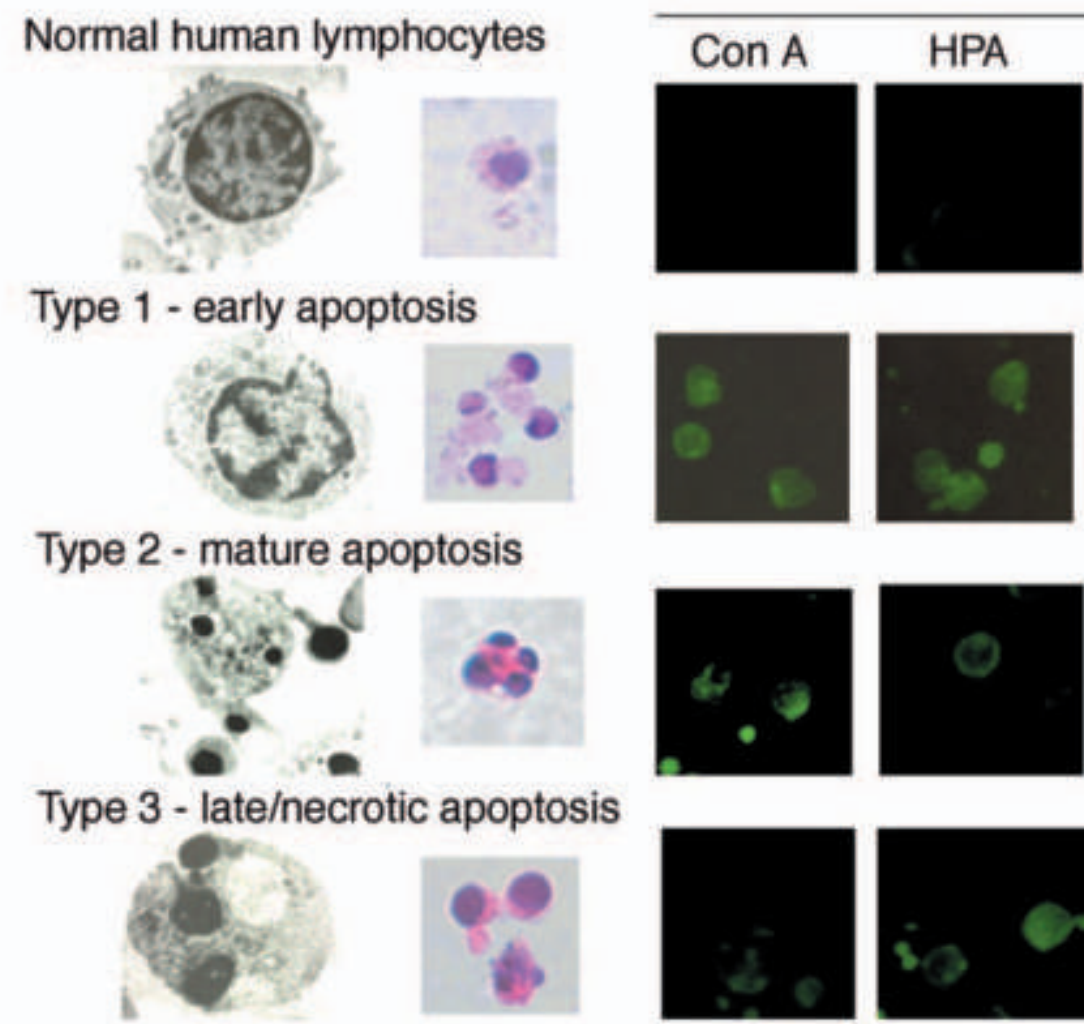

Lectins-FITC
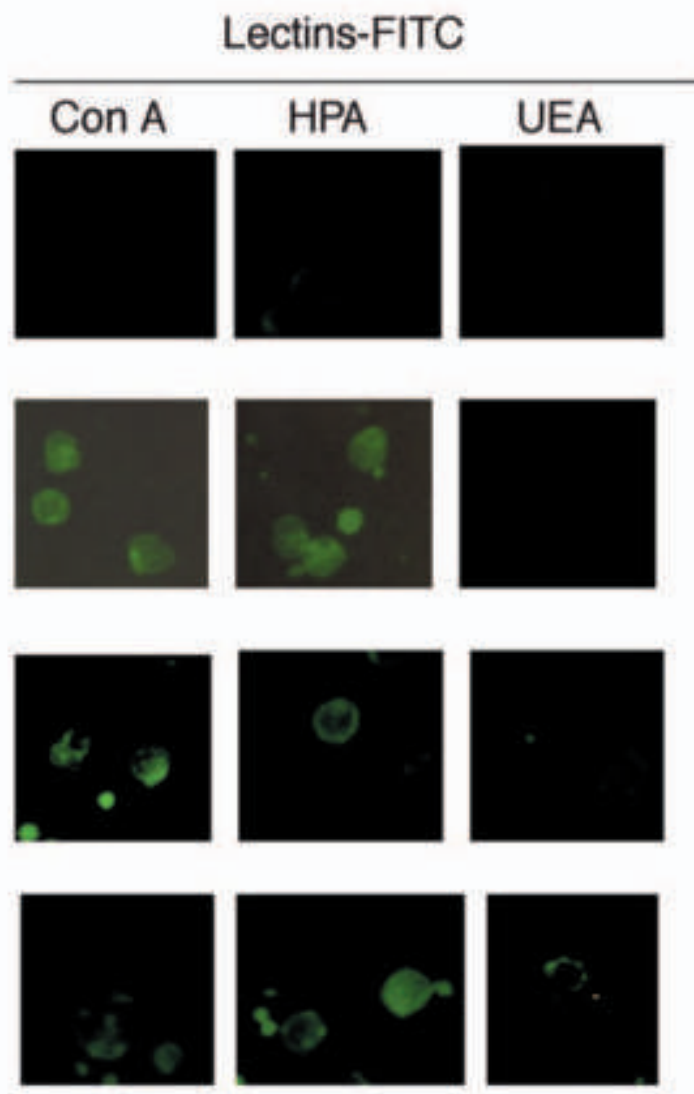
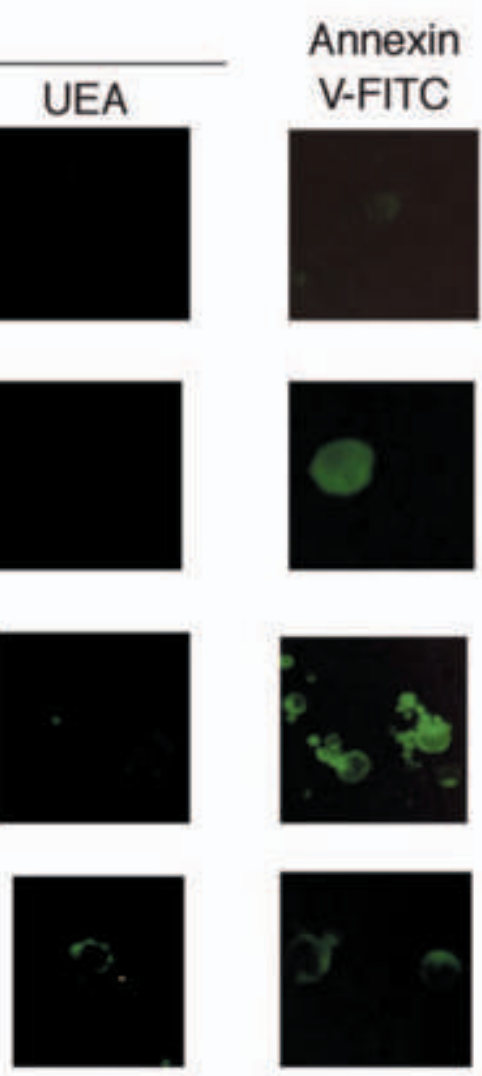

Figure 1. Morphologies and cell surface properties of apoptotic human lymphocytes type 1, 2 and 3. In type 1 or early apoptosis no morphological modifications are observed in the majority of the cell population. Modifications of the cell surface include scarce exposure of AnnexinV, ConA and HPA binding sites. Fucose residues are absent from the surface. In type 2 or mature apoptosis modifications of nucleus (fragmentation) and cell shape (blebbing) are observed. Cell surface is largely modified: exposure of PS and sugar residues. In type 3 or late/necrotic apoptosis; most of the apoptotic cells undergo secondary necrosis. Cell surface modifications show a decreased expression of PS and the appearance of sugar residues such as fucose, absent from the surface of early apoptotic lymphocytes. Concanavalin-A (Con-A, $40 \mathrm{mg} / \mathrm{mL}$, mannose), Helix pomatia (HPA, $60 \mathrm{mg} / \mathrm{mL}$, N-acetylgalctosamina), Ulex europaeus (UEA, $40 \mathrm{mg} / \mathrm{mL}$, $\alpha$-fucose), Annexin V (20 mM, phosphatydilserine). 
Table 1. Percentage of type 1,2 and 3 apoptosis among human lymphocytes incubated with CHX 10-2M for $24 \mathrm{hrs}$.

\begin{tabular}{lccc}
\hline \hline & $0 / 6$ hrs & $6 / 18$ hrs & $18 / 24$ hrs \\
\hline Type 1 & 94,5 & 15 & 10 \\
Type 2 & 5 & 65 & 35 \\
Type 3 & 0.5 & 20 & 55 \\
\hline
\end{tabular}

One representative experiment out of three performed is reported.

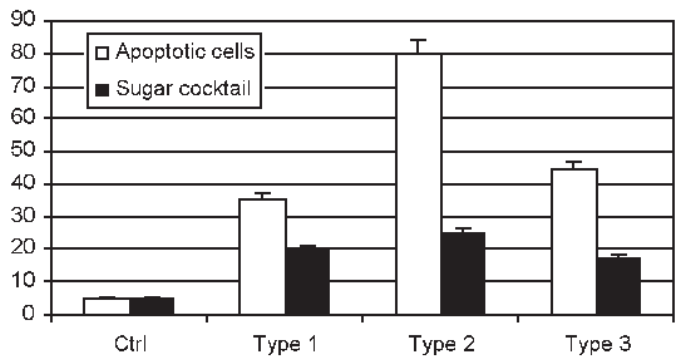

Figure 2. Percentage of human lymphocytes (control, type 1, 2 and 3) retained by mouse sinusoidal liver cells. Mouse livers were in situ injected with control or apoptotic lymphocytes as described in the materials and methods section. The highest liver recognition was achieved with type 2 apoptosis lymphocytes and the lowest with type 1 . The addition of a sugar cocktail prevented the recognition of apoptotic cells to an extent related to the type of apoptosis.

population showed no sign of apoptotic morphological modifications, although some cells show mild margination of chromatin. Very few cells show the stereotypical apoptotic morphology. Modifications of the cell surface (i.e. increased PS and sugar residue expression) were detected, independently of the nuclear modifications.

Type 2 or mature apoptosis. The majority of cells showed modifications of nucleus and cell shape (blebbing). The cell surface was highly modified, with extensive exposure of PS and sugar residues.

Type 3 or late/necrotic apoptosis. Most of the apoptotic cells underwent secondary necrosis. Cell surface modifications showed a decreased exposure of PS and abundant expression of sugar residues such as fucose, absent from the surface of early apoptotic lymphocytes. When surface modifications are considered, differences were observed between human and rat lymphocytes and between rat and mouse lymphocytes, human and mouse lymphocytes being similar (Table 2).
Rat lymphocytes

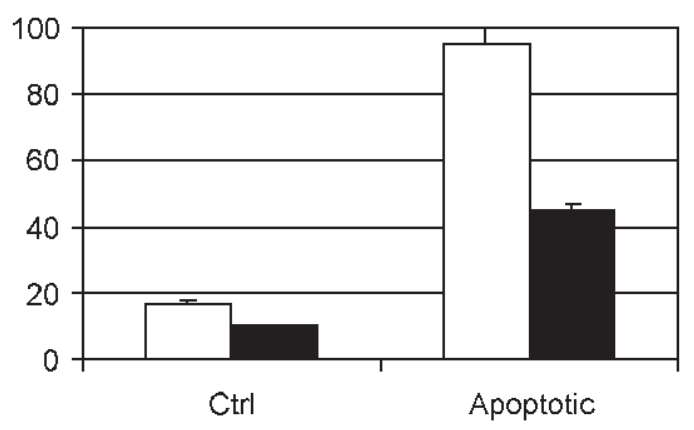

Human lymphocytes

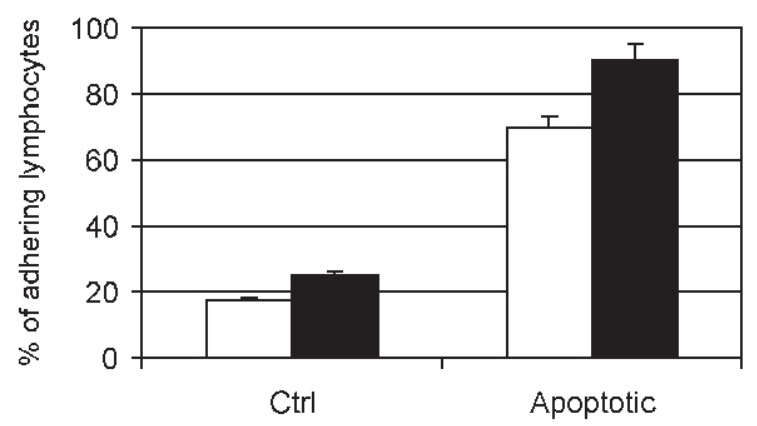

Mouse lymphocytes

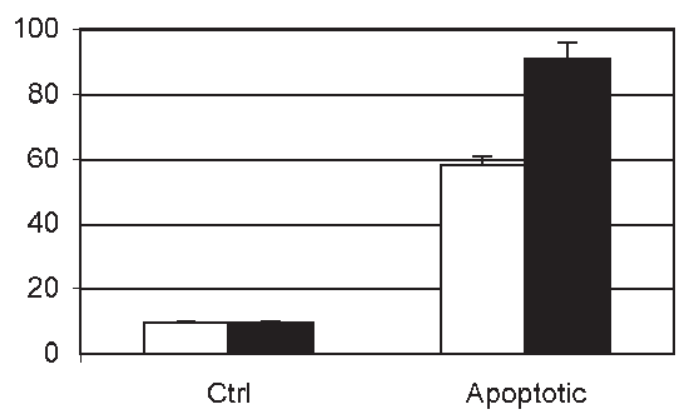

$\square$ Rat liver sinusoidal cells

Mouse liver sinusoidal cells

Figure 3. Percentage of apoptotic lymphocytes from rat (panel a), human (panel b) and mouse (panel c) adhering to sinusoidal cells of rat and mouse livers. The highest recognition rate was obtained when homologous (i.e. rat) apoptotic lymphocytes were injected into rat liver. The lowest recognition rate was obtained when rat apoptotic lymphocytes where injected into mouse liver.

Liver injected with apoptotic lymphocytes enriched for type 1,2 or 3 apoptosis recognized and internalized apoptosing cells, with an efficiency 
Rat lymphocytes

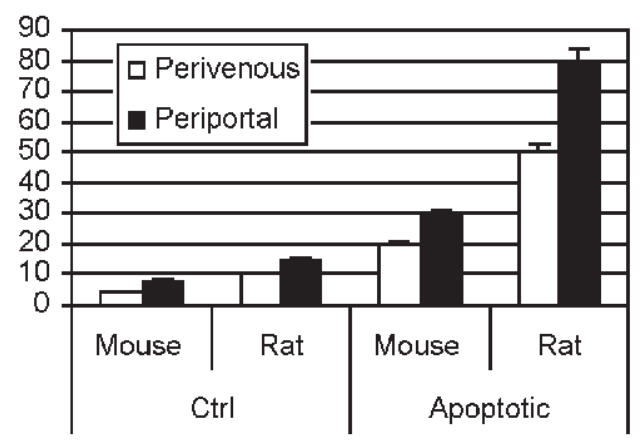

Human lymphocytes

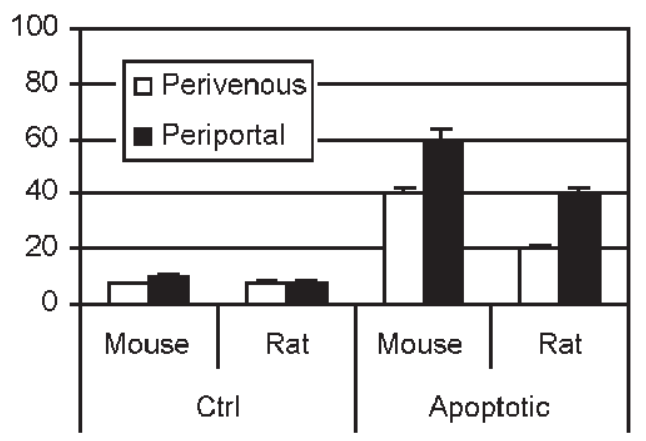

Figure 4. Percentage of apoptotic lymphocytes from human and rat adhering to periportal and perivenous sinusoidal cells of rat and mouse livers. Liver sinusoids of periportal zone retained higher amounts of apoptotic lymphocytes than sinusoidal cells of perivenous tracts, due to their high levels of mannose- and galactose-specific receptors. Homologous recognition was always higher than heterologous. directly dependent on the stage of the apoptotic process (Figure 2). Interestingly, the maximum inhibition of recognition (by using a sugar cocktail) was achieved with type 2 - mature apoptosis. The data in Figure 2 in fact, suggest that type 1 or type 3 apoptotic lymphocytes are mainly recognized by liver cells by means of molecules differing from lectin-like receptors.

\section{Species specificity of apoptotic lymphocyte recognition by the liver}

The in situ injection of apoptotic lymphocytes from rat or human to rat or mouse liver is reported in Figures 3 and 4. The data show that the highest recognition rate was obtained when homologous (i.e. rat) apoptotic lymphocytes were injected into rat liver. Sinusoidal rat liver cells (SEC) retained more than $90 \%$ of the injected homologous lymphocytes. Conversely, $70 \%$ of the injected apoptotic human lymphocytes and $45 \%$ of injected rat lymphocytes were recognized respectively by rat and mouse sinusoidal endothelial cells (SEC). Similar results were obtained when mouse apoptotic lymphocytes were injected into mouse liver circulation (Figure 3, panel C). Human apoptotic lymphocytes were better recognized by the mouse liver SECs than by the rat liver SECs ( $87 \%$ as against $70 \%$ ).

The recognition of apoptotic lymphocytes was, in all cases, mediated by specific receptors, i.e. galactose and mannose, which recognized the surface modifications. The experiments reported in Figure 4 support the presence of sugar specific receptors able to recognize apoptotic lymphocytes. In fact, the liver sinusoid tracts (periportal) that express

Table 2. Major differences and/or similarities among human, rat and mouse apoptotic lymphocytes induced to apoptotis by $10^{-2} \mathrm{M}$ CHX.

\begin{tabular}{|c|c|c|c|c|c|c|c|c|c|}
\hline & \multicolumn{3}{|c|}{ Human } & \multicolumn{3}{|c|}{ Rat } & \multicolumn{3}{|c|}{ Mouse } \\
\hline & Early & Mature & Late & Early & Mature & Late & Early & Mature & Late \\
\hline Chromatin condensation & -+ & ++ & +++ & -++ & +++ & +++ & -+ & ++ & +++ \\
\hline Nucleus fragmentation & - & ++ & + & - & + & - & - & ++ & + \\
\hline PS & ++ & ++++ & +++ & + & +++ & ++ & ++ & ++++ & +++ \\
\hline ConA binding sites & ++ & +++ & +++ & + & ++ & ++ & + & +++ & ++ \\
\hline HPA binding sites & - & ++ & ++ & - & + & ++ & - & +++ & ++ \\
\hline UEA binding sites & - & -+ & ++ & - & - & + & - & +- & + \\
\hline Blebs* & - & +++ & ++ & - & - & -+ & - & + & + \\
\hline
\end{tabular}

+Increase; -absence; *cytoplasmatic. 
high levels of mannose- and galactose-specific receptors are able to retain a high amount of apoptotic lymphocytes. However, homologous recognition was always higher than heterologous. In addition, normal control lymphocytes were always retained by liver (rat or mouse) to a very low extent (less than $10 \%$ of injected lymphocytes). Again, a mixture of sugars (80 mM N-acetyl-D-glucosamine, $\mathrm{N}$-acetyl-D- galactosamine, fucose, mannose), injected simultaneously with the apoptotic cells, reduced the recognition of apoptotic lymphocytes by about $70-90 \%$. However, $10-20 \%$ of injected apoptotic cells still bound to the sinusoids (Figure 2 ); the percentage of non-inhibited binding was constant in type 1,2 or 3 apoptosis, and higher in the inhibition experiments on homologous interactions (rat/rat and mouse/mouse), thus indicating that the recognition of apoptotic lymphocytes involves receptors and molecules different from lectin-like receptors and sugar residues. Moreover, the drastically reduced efficiency of recognition of human or mouse apoptotic lymphocytes when injected into rat liver further suggests the involvement of species-specific antigens for the recognition of apoptotic cells.

\section{Discussion}

A myriad of phagocyte receptors have been implicated in the recognition of apoptotic cells, among them a PS receptor, and yet few ligands other than PS have been identified on the apoptotic cell surface, besides sugar residues (Schlegel and Williamson, 2001). This is supported by the data here reported, clearly indicating that liver apoptotic recognition involves more than one type of ligand/receptor interaction. The modifications on the cell surface of apoptotic cells are widespread andconserved in evolution, in particular the exposure of PS. In fact, apoptosis and the associated exposure of PS on the cell surface is probably over 600 million years old and therefore the conservation of surface modifications (Schlegel and Williamson, 2001) in different Mammalian species as shown here for mouse, rat and human is not surprising. On the other hand, evolution has appropriated aspects for other purposes, as is the case for liver lectin-like receptors. Our data are in agreement with the above-mentioned studies: the type of surface modification (PS and sugar exposure) is the same in different cell types and in the same cell types of different species. However, during evolu- tion, irrespective of cell type, peculiarities in the pattern of cell surface modifications have been introduced, i.e. timing of exposure, quality and quantity. In any case, surface modifications of the apoptotic lymphocytes are the prerequisite for their recognition and subsequent engulfment (Savill, 1998; Savill and Fadok, 2000; Schlegel and Williamson, 2001; Fadok et al. 2001 ).

From the in situ adhesion experiments on enriched populations of type 1,2 or 3 apoptotic lymphocytes, it appears that the efficiency of recognition and subsequent engulfment is a balance of quality, quantity and timing of exposure of PS and sugar residues on the apoptotic cells and the amount of receptors present on the phagocytic cells. The accumulation of apoptotic lymphocytes was not homogenous in the liver: periportal zones retained much more than perivenous ones. In fact, the different recognition of apoptotic lymphocytes by the SECS of periportal and perivenous liver zones, has been attributed to the different amount of receptors for mannose and galactose residues expressed on the cell surface (Dini and Carlà 1998).

All the inhibition experiments suggest that recognition of apoptotic lymphocytes is mainly mediated by lectin-like receptors co-adjuvated by other molecules for type 2 apoptosis; conversely, the recognition of type 1 or type 3 apoptotic lymphocytes is mainly mediated by receptors different from lectinlike ones, probably scavenger receptors type $A$ and B (Shiratsuchi et al. 1999; Platt et al. 1996). Recent data indicates that most of the receptors that have been indicated for the recognition of apoptotic cells, including the liver lectin receptors, may provide the strong adhesion needed to increase the likelihood of contact between the PS receptor and its phospholipid ligand, which is required for uptake (Fadok et al. 2001). Given that for a high rate of adhesion the species is important as well, as the experiments on heterologous adhesion have indicated, a major candidate, apart from the phospholipid ligand and its receptor, is species-specific antigen molecules. But why is rapid self-recognition of apoptotic cells necessary? One possible explanation could be lie in the distinct immunological function of the liver; probably the higher rate of recognition between homologous apoptotic cells and phagocytes is a mechanism for specifically accumulating in the liver a large number of apoptotic cells, that in turn, are partially responsible for the liver immune tolerance (Sun and Shi 2001). Another 
explanation could be the risk linked to the presence in the circulation of apoptotic cells, which can be a source of autoantigens (Rodenburg et al. 2000; Rovere et al. 1999; Lorenz et al. 1997). Indeed, it is known that in systemic autoimmunity their presence in the blood is increased (Kovacs et al. 1996). Further supporting the notion that apoptotic cells act as supplier of autoantigens are the data regarding immunization with apoptotic murine thymocytes (Mevorach et al. 1998). However, the signals transduced from the phagocyte to lymphocyte or vice-versa are still unknown. On the other hand, if apoptotic cells are present in large numbers, overwhelming the phagocytic capacity, or if recognition is not efficient, the apoptotic cells will accumulate and undergo secondary necrosis. Such necrotic cells release danger signals, which stimulate the immune system.

In conclusion our experiments suggests that livers recognize and internalize apoptosing cells by means of galactose- and mannose- specific receptors, with an efficiency directly dependent on the stage of the apoptotic process (i.e. type 1 (early), 2 (mature) or 3 (late/necrotic) apoptosis), and on the presence of species-specific antigens for enhancing the removal rate.

\section{References}

Desmet VJ. Introduction:organizational principles. In The Liver: biology and pathobiology, $3^{\text {rd }}$ ed. Raven Press: New York; 1994. p. 3-14.

Dini, L, Carlà EC. Hepatic sinusoidal endothelium heterogeneity with respect to the recognition of apoptotic cells. Exp Cell Res 1998; 240:388-93.

Dini L. Recognizing death: liver phagocytosis of apoptotic cells. Eur J Histochem 2000;44:217-27.

Dini L, Pagliara P, Carlà EC. Phagocytosis of apoptotic cells by liver: a morphological study. Microsc Res Techn 2002;57:530-40.

Crispe IN, Dao T, Klugewitz K, Mehal WZ, Metz DP. The liver as a site of T-cell apoptosis: graveyard or killing field? Immunol Rev 2000; 174:47-62

Fadok VA, Xue D, Henson P. If phosphatidylserine is the death knell, a new phosphatidylserine-specific receptor is the bellringer. Cell Death Differ 2001;8:582-7.

Kovacs B, Vassilopoulos D, Vogelgesang SA, Tsokos GC. Defective CD3mediated cell death in activated T cells from patients with systemic lupus erythematosus: role of decreased intracellular TNF-alpha. Clin Immunol Immunopathol 1996;81:293-302.

Lorenz HM, Grunke M, Hieronymus T, Hermann M, Kuhnel A, Manger $\mathrm{B}$, et al. In vitro apoptosis and expression of apoptosis-related molecules in lymphocytes from patients with systemic lupus erythematous and other autoimmune diseases. Arthritis Rheum 1997;40: 306-17.

Mevorach D, Zhou JL, Song X, Elkon KB. Systemic exposure to irradiated apoptotic cells induces autoantibody production. J Exp Med 1998;188:387-92.

Platt N, Suzuki H, Kurihara Y, Kodama T, Gordon S. Role for the class A macrophage scavenger receptor in the phagocytosis of apoptotic thymocytes in vitro. Proc Natl Acad Sci USA 1996;93:12456-60.

Rodenburg RJ, Raats JM, Pruijn GJ, van Venrooij WJ. Cell death: a trigger of autoimmunity? Bioessays 2000;22:627-36.

Rosen A, Casciola-Rosen L. Autoantigens as substartes for apoptotic proteases: implications for the pathogenesis of systemic autoimmune disease. Cell Deth Differ 1999;6:6-12.

Rovere $P$, Sabbadini MG, Vallinoto $C$, Fascio $U$, Zimmermann VS, Bondanza $A$, et al. Delayed clearance of apoptotic lymphoma cells allows cross-presentation of intracellular antigens by mature dendritic cells. J Leukoc Biol 1999;66:345-9.

Savill J. Apoptosis. Phagocytic docking without shocking. Nature 1998;392:442-3.

Savill J, Fadok V. Corps clearance defines the meaning of cell death. Nature 2000;407:784-7.

Schlegel RA, Williamson P. Phosphatidylserine, a death knell. Cell Death Differ 2001;8:551-63.

Shiratsuchi A, Kawasaki Y, Ikemoto M, Arai H, Nakanishi Y. Role of class B scavenger receptor type I in phagocytosis of apoptotic rat spermatogenic cells by Sertoli cells. J Biol Chem 1999;274:59018.

Smedsrot B, Pertoft H, Gustafson S, Laurent TC. Scanvenger functions of the liver endothelial cell. Biochem J 1990;266:313-27.

Starzel TE, Demetris AJ, Van Thiel D. Liver transpalantation. N Engl J Med 1989;321:1092-9.

Sun EW, Shi YF. Apoptosis: the quiet death silences the immune system. Pharmacol Ther 2001;92:135-45. 
A. Chionna et al. 